\section{Dealing with post-dural puncture headache - is it different in obstetrics?}

$\mathrm{P}$ OST-DURAL puncture headache (PDPH) won't go away. The message is clear: the incidence and severity of PDPH is related to the size of the hole in the dura. This type of headache is more common in younger people, and more prevalent in women than in men. It occurs more frequently in people who are prone to headaches. Pregnancy per se does not increase the relative risk of PDPH. The high incidence of spinal headache in obstetric patients may be explained by age and sex. ${ }^{1}$

Numerous studies and reports have chronicled the benefits of using small gauge, pencil point needles when performing deliberate lumbar puncture for anaesthesia or diagnostic procedures. ${ }^{2}$ Young obstetric patients frequently undergo spinal anaesthesia for Caesarean section, and anaesthetists now use needles designed to minimise the incidence of PDPH, which remains around $1 \%$. This is considered an acceptable risk.

Obstetric patients are unique, however, in that they are likely to suffer accidental dural puncture with a large bore epidural needle designed to introduce catheters into the epidural space. The incidence of this complication varies with the experience of the anaesthetist, and is $0.5-2.5 \%$ in teaching hospitals. ${ }^{3}$ When unintentional dural puncture occurs, 30 to $70 \%$ of the parturients get the headache. The variance is explained by the direction of the needle in relation to the dural fibres when the entry occurs. ${ }^{4}$ If the epidural needle is introduced parallel to the longitudinal dural fibres, the incidence of PDPH is much less.

When PDPH develops in the obstetric patient, the morbidity is considerable. The new mother is incapacitated, she may be unable to get out of bed to care for her baby or herself. The cost of health care increases, because she may need to stay in hospital longer than planned.

Treatment options for the condition are not particularly satisfactory. Pharmacological treatment is variable at best. Intravenous or oral caffeine give variable results. Epidural or intravenous saline infusions have not been shown to be of much use. Bed rest is no longer recommended. Recent reports on the success- ful use of subcutaneous sumatriptan to treat the headache merits further study. ${ }^{5}$

If the mother is too distressed to get up and move, the epidural blood patch is still the best treatment, although it is not as successful as previously thought. Early reports suggested that epidural blood patches were $90 \%$ successful in curing the headache. It is now known that the headache recur in $15-30 \%$ of patients even after a successful initial patch, and permanent cure is only achieved in about $60 \%$ of patients after one blood patch. ${ }^{6}$ Repeating the blood patch once may improve the headache in another $20 \%$, but more than two blood patches is not recommended.

Blood patching is not without side effects and complications. Patients may be reluctant to undergo this treatment because another epidural needle must be inserted, with the risk of repeat dural puncture. Back pain is common, perhaps due to tracking of blood back into the subcutaneous fat. ${ }^{7}$ The risk of infection, albeit rare, should not be discounted. A recent report described postpartum dural venous sinus thrombosis after a blood patch. ${ }^{8}$

A number of legal claims have resulted from PDPH in Canada. Although all have been dismissed, the incidence of this complication in anaesthetic practice is high enough to warrant warning all patients that it can occur. This will decrease costs associated with medicolegal defence.

Since treatment of PDPH, a self limiting condition, may be unsatisfactory, ineffective or risky, any prophylactic measure that can consistently prevent the headache is worth investigating.

In this issue, Dennehy and Rosaeg ${ }^{9}$ report that in three cases, insertion of an intrathecal catheter after accidental dural puncture, and leaving that catheter in situ for more than $12 \mathrm{hr}$, was effective in preventing PDPH. They discuss two previous reports with the same findings (Dennehy's References \#5 and 14). Cohen et al. left the catheter in place after Caesarean section, and used it to provide intrathecal patient controlled pain relief with fentanyl and bupivacaine. That, in itself, 
might have prevented PDPH. Denny's study was carried out using continuous spinal anaesthesia in surgical patients of all ages. While only one young man in this series developed PDPH, no conclusions can be drawn regarding the incidence of PDPH in young women, since age differentials are not discussed.

Two papers contradict the findings in Dennehy's report. Norris and Leighton prospectively inserted spinal catheters into one group of parturients who had unintentional dural puncture, and omitted the catheter in the other group. The catheter stayed in for at least two hours. They found no difference in the incidence of headache or the necessity for blood patch in the two groups. ${ }^{3}$ Another paper discussed leaving a 20-gauge catheter in situ intrathecally for 12-24 hours after spinal anaesthesia for orthopaedic surgery. The patients were older than most pregnant women. There was no difference in the incidence of PDPH whether the catheter was left in or taken out immediately after surgery. ${ }^{10}$ In both these studies, the authors stress that the 18-gauge epidural needles were inserted with the bevel parallel to the dural fibres, thus reducing the expected incidence of PDPH. Dennehy does not mention the direction of the needle bevel. Two of their patients received intrathecal fentanyl, and one patient was obese: both factors that may decrease the incidence of headache.

If one postulates that leaving the catheter in situ reduces the incidence of $\mathrm{PDPH}$, what is the explanation? Preventing the headache may be related to an inflammatory reaction and deposition of fibrin where the catheter enters the dura, as Dennehy et al. discuss. However, in the rat studies referred to in their paper, the catheters were left in place for 19-21 days. The fibrin theory, while attractive, is still unproven in clinical practice in obstetric patients.

It may be a good idea to insert the catheter intrathecally and use it to provide superior labour analgesia or anaesthesia for Caesarean section. Extraordinary care must be taken when using the intrathecal catheter. It is an epidural catheter, and unless one labels it "intrathecal" and informs all staff who may provide labour analgesia, mistakes can easily be made. The wrong drug, concentration or volume may be given, with predictable, but disastrous results.

Many anaesthetists leave catheters in position after unintentional dural puncture. In my experience some patients develop PDPH, others do not. The three cases described in this journal neither prove nor disprove that leaving the catheter in for several hours is an effective prophylactic technique for prevention of PDPH. What is needed is a larger study designed to compare the incidence of PDPH in mothers with catheters left in for less than two hours and longer than 12 hours, where the drugs used are comparable.

\section{Céphalée post ponction dure-mère : est-ce différent en obstétrique ?}

Les céphalées post ponction de la dure-mère (CPPDM) ne disparaîtront pas. Le message est clair: l'incidence et la sévérité de ces céphalées dépend de la grosseur du trou dans la dure-mère. Ce type de céphalée survient plus fréquemment chez les jeunes gens, chez la femme plus que chez l'homme et chez les gens prédisposés aux céphalées. La grossesse en soi n'accroît pas le risque. L'incidence élevée de ce type de céphalée en obstétrique s'explique donc par l'âge et le sexe !

De nombreuses études et publications ont établi les avantages à utiliser des aiguilles de petit calibre avec pointe de crayon pour la ponction lombaire lors d'anesthésies ou de procédures diagnostiques. ${ }^{2}$ On pratique souvent des rachianesthésies pour césarienne chez des jeunes femmes, et les anesthésistes utilisent maintenant des aiguilles spéciales destinées à diminuer l'incidence des CPPDM, cette incidence demeurant aux environs de $1 \%$, ce qui est considéré un risque acceptable.

Les parturientes représentent une population particulière appelée à subir des ponctions accidentelles de la dure mère avec des aiguilles épidurales de gros calibre nécessaires pour la réalisation d'épidurales continues. L'incidence de cette complication varie avec l'expérience de l'anesthésiste et est de 0,5-2.5\% dans les hôpitaux d'enseignement. ${ }^{3}$ Lors de ponction de dure-mère non intentionnelle, la céphalée survient dans 30 à $70 \%$ des cas. L'écart dans l'incidence s'explique par l'orientation de l'aiguille par rapport aux fibres de la dure-mère au moment de la ponction. ${ }^{4}$ Lorsque l'aiguille pénètre de façon parallèle aux fibres durales qui sont longitudinales, l'incidence de céphalée est alors plus basse.

Lorsque la CPPDM apparaît chez la parturiente, la morbidité est considérable. La nouvelle maman est incapacitée, elle peut être incapable de se lever pour s'occuper d'elle-même ou de son bébé. Les coûts de santé augmentent car le séjour hospitalier peut s'allonger. 
Les options thérapeutiques ne sont pas très satisfaisantes. Au mieux, le succès du traitement pharmacologique est variable. La caféine orale ou intraveineuse donne des résultats variables. Les infusions de salin tant intraveineuses qu'épidurales n'ont pas donné beaucoup de résultats. Le repos au lit n'est plus recommandé. Des rapports récents concernant les succès du sumatriptan par voie sous-cutanée méritent d'autres études. ${ }^{5}$

Si la nouvelle maman est trop symptomatique pour se lever et bouger, le pansement de sang épidural est toujours le meilleur traitement, même s'il n'est pas aussi efficace qu'on le croyait antérieurement. Les premières études faisaient état de succès de l'ordre de $90 \%$. Maintenant, on sait que les céphalées réapparaissent chez 15 à $30 \%$ des patientes même après un pansement initial efficace, et que la cure permanente n'est atteinte que chez $60 \%$ des patientes après un pansement épidural. ${ }^{6} \mathrm{Si}$ on répète le pansement, on améliore la céphalée chez un autre $20 \%$, mais plus de deux pansements n'est pas recommandé.

Le pansement de sang épidural n'est pas dénué d'effets secondaires et de complications. Les patientes peuvent être hésitantes à subir cette procédure parce qu'il faut refaire une épidurale, avec le risque d'une nouvelle ponction de la dure-mère. La douleur lombaire est fréquente, probablement due à l'échappement du sang dans la graisse sous-cutanée en suivant le trajet de la ponction ? Le risque d'infection, même s'il est rare, ne doit pas être oublié. Un rapport récent fait état d'une thrombose d'un sinus veineux dural survenu en postpartum à la suite d'un pansement épidural. ${ }^{8}$

Les CPPDM ont entrainé un certain nombre de poursuites légales au Canada. Même si toutes ont été rejetées, l'incidence de cette complication dans la pratique anesthésique est suffisamment élevée pour nécessiter d'en aviser toutes les patientes. Ceci diminuera les coûts associés à la défense médico-légale.

Comme le traitement de la CPPDM, condition limitée par elle-même, peut être insatisfaisant, inefficace ou même risqué, toute mesure prophylactique pouvant prévenir de façon efficace la céphalée vaut d'être investiguée.

Dans ce numéro, Dennehy et Rosaeg9 rapportent que, dans trois cas, l'insertion du cathéter après ponction accidentelle de la dure-mère et le fait de laisser ce cathéter en place pour plus de 12 heures a été efficace pour prévenir la CPPDM. Les auteurs discutent deux articles antérieurs faisant état des mêmes résultats (références 5 et 14 de Dennehy). Cohen et al ont laissé le cathéter en place après césarienne, l'utilisant pour fournir une analgésie sous-arachnoïdienne contrôlée par la patiente et basée sur l'utilisation de bupivacaine et de fentanyl. Ce régime, en soi, peut avoir prévenu la CPPDM. L'étude de Denny portait sur l'analgésie rachidienne continue chez des patients de tout âge. Bien que seul un jeune homme ait développé une CPPDM, aucune conclusion ne peut être inférée à des CPPDM chez des jeunes femmes, étant donné que la relation avec l'âge n'est jamais discutée.

Deux études contredisent les trouvailles de Dennehy. Norris et Leighton, de façon prospective, chez des patientes ayant eu une ponction accidentelle de la duremère, insérèrent un cathéter sous-arachnoïdien chez la moitié des sujets, omettant le cathéter chez l'autre moitié. Les cathéters demeurèrent en place pour au moins 2 heures. Les auteurs ne trouvèrent aucune différence quant à l'incidence de céphalée et quant au besoin de pansement épidural. ${ }^{3}$ Une autre étude discutait de l'intérêt de laisser un cathéter de calibre 20 en place en sous arachnoïdien pour 12-24 heures après rachianesthésie en orthopédie. Les patients étaient plus âgés que la majorité des parturientes. Il n'y avait aucune différence dans l'incidence des céphalées, que le cathéter soit laissé en place ou retiré immédiatement après la chirurgie. ${ }^{10}$ Dans ces deux études, les auteurs insistaient sur le fait que le biseau des aiguilles épidurales de calibre 18 était inséré parallèlement aux fibres durales, réduisant ainsi l'incidence attendue des céphalées. Dennehy ne mentionne pas l'orientation du biseau de l'aiguille. Deux patients ont reçu du fentanyl intrathécal et un patient était obèse : ces deux facteurs peuvent diminuer l'incidence des céphalées.

Si on postule que le fait de laisser en place le cathéter en position sous-arachnoïdienne diminue l'incidence des céphalées, quelle est l'explication ? La prévention de la céphalée peut être due à une réaction inflammatoire et à une déposition de fibrine où le cathéter pénètre la duremère, tel que Dennehy et al le discutent. Cependant, dans les études chez le rat auxquelles ils réferent, les cathéters étaient laissés en place pour 19 à 21 jours. La théorie de la fibrine, bien qu'attrayante, demeure non prouvée en pratique clinique chez des patientes obstétricales.

C'est peut-être une bonne idée d'insérer le cathéter en sous-arachnoïdien et de l'utiliser pour procurer une qualité supérieure d'analgésie pour le travail ou d'anesthésie pour la césarienne. Un soin extraordinaire doit être déployé lors de l'utilisation de cathéters sous arachnoïdiens. Il s'agit d'un cathéter épidural, et à moins de clairement l'identifier «intrathécal» et d'en informer tous les membres du personnel responsable de l'analgésie durant le travail, des erreurs peuvent facilement se produire. La mauvaise médication, de par sa concentration ou son volume, peut être administrée avec des résultats prévisibles mais désastreux.

Plusieurs anesthésistes laissent un cathéter en place après ponction de la dure-mère non intentionnelle. 
Dans mon expérience, certaines patientes développent une céphalée, d'autres non. Les 3 cas rapportés dans ce numéro ne prouvent, pas plus qu'il n'infirment, que le fait de laisser un cathéter en place pour plusieurs heures est une technique prophylactique efficace dans la prévention des CPPDM. Ce qu'il faut, c'est une étude plus vaste comparant l'incidence des céphalées chez les parturientes où le cathéter est laissé en place pour moins de 2 heures et pour plus de 12 heures, et où les médications utilisées sont comparables.

\section{References}

1 Morewood GH. A rational approach to the cause, prevention and treatment of postdural puncture headache. Can Med Assoc J 1993; 149: 1087-93.

2 Halpern S, Preston R. Postdural puncture headache and spinal needle design. Metaanalyses. Anesthesiology 1994; 81: 1376-83.

3 Norris $M C$, Leighton $B L$. Continuous spinal anesthesia after unintentional dural puncture in parturients. Reg Anesth 1990; 15: 285-7.

4 Norris $M C$, Leighton BL, DeSimone CA. Needle bevel direction and headache after inadvertent dural puncture. Anesthesiology 1989; 70: 729-31.

5 Carp $H$, Singh PJ, Vadhera R, Jayaram A. Effects of the serotonin-receptor agonist sumatriptan on postdural puncture headache: report of six cases. Anesth Analg 1994; 79: 180-2.

6 Taivainen T, Pitkanen M, Tuominen M, Rosenberg PH. Efficacy of epidural blood patch for postdural puncture headache. Acta Anaesthesiol Scand 1993; 37: 702-5.

7 Beards SC, Jackson A, Griffiths AG, Horsman EL. Magnetic resonance imaging of extradural blood patches: appearances from $30 \mathrm{~min}$ to $18 \mathrm{~h}$. $\mathrm{Br} J$ Anaesth 1993; 71: 182-8.

8 Borum SE, Naul LG, McLeskey CH. Postpartum dural venous sinus thrombosis after postdural puncture headache and epidural blood patch. Anesthesiology 1997; 86: 487-90.

9 Dennehy KC, Rosaeg OP. Insertion of intrathecal catheter during labour reduces the risk of post-dural puncture headache. Can J Anaesth 1997; 44: 42-5.

10 Liu N, Montefiore A, Kermarec N, Rauss A, Bonnet F. Prolonged placement of spinal catheters does not prevent postdural puncture headache. Reg Anesth 1993; 18: 110-3. 